\title{
EMBEDDING A PARTIALLY ORDERED RING IN A DIVISION ALGEBRA
}

\author{
BY \\ WILLIAM H. REYNOLDS
}

\begin{abstract}
D. K. Harrison has shown that if a ring with identity has a positive cone that is an infinite prime (a subsemiring that contains 1 and is maximal with respect to avoiding -1 ), and if the cone satisfies a certain archimedean condition for all elements of the ring, then there exists an order isomorphism of the ring into the real field. Our main result shows that if Harrison's archimedean condition is weakened so as to apply only to the elements of the cone and if a certain centrality relation is satisfied, then there exists an order isomorphism of the ring into a division algebra that is algebraic over a subfield of the real field. Also, Harrison's result and a related theorem of D. W. Dubois are extended to rings without identity; in so doing, it is shown that order isomorphic subrings of the real field are identical.
\end{abstract}

Several authors have considered certain subsemirings of a ring with identity that have some properties in common with the pointwise positive cones of rings of bounded real-valued functions; in so doing, they have obtained representations of such rings as subrings of rings of continuous real-valued functions on compact spaces or as subrings of the real field. For some examples, see [1, Theorem 1], [3, Proposition 7], and [6, p. 317]. By introducing similar concepts in abelian groups, C. W. Kohls [5] has given simpler more direct proofs of Harrison's and Krivine's results as well as obtaining a representation theorem for groups.

It was hoped that [3, Proposition 7] could be extended so as to obtain representation of a certain class of rings as subrings of the division ring of real quaternions, for it is noted that many subrings of the quaternions satisfy our hypotheses. What we actually obtain is representation of such rings as subrings of division algebras that are algebraic over subfields of the reals. However, if the rings are commutative, the desired result follows; that is, they are then isomorphic to subrings of the complex field. In the general case, we can show that the semiring of nonnegative reals satisfies our hypotheses as a subsemiring of a $\operatorname{ring} A$ iff $A$ is isomorphic to either the real field, the complex field, or the quaternions. This is quite analogous to Frobenius' theorem [8] which states that an algebraic real division algebra is isomorphic to one of these; indeed we use his theorem in our proof.

The real field is denoted by $R$, the subsemiring of nonnegative reals by $R^{+}$, and the set of positive integers by $N$. If $S, T$ are subsets of a ring $A$, we write $S-T$ for

Received by the editors September 11, 1970 .

AMS 1969 subject classifications. Primary 0620, 0685, 1646; Secondary 4625, 1340.

Key words and phrases. Harrison infinite prime, ordered ring, representation, real-valued continuous function, compact space, algebraic division algebra.

Copyright (C) 1971, American Mathematical Society 
$\{s-t: s \in S, t \in T\}, S T$ for $\{s t: s \in S, t \in T\}$, and $S \backslash T$ for $\{s \in S: s \notin T\}$. In a ring $A$, we assume the convention $a^{0} b=b a^{0}=b$ for every $a, b \in A$. Given a topological space $X, C(X)$ is the ring of all continuous real-valued functions on $X$ with pointwise addition, multiplication and order. Much of our terminology is similar to that found in [5].

This paper is part of a dissertation directed by Professor Carl W. Kohls. The dissertation was submitted to Syracuse University in partial fulfillment of the requirements for the degree of Doctor of Philosophy. Much of the work was supported by a National Science Foundation Traineeship.

1. Rings without identity. We here extend [1, Theorem 1] and [3, Proposition 7] to rings without identity.

Definitions. Let $u$ be a nonzero element in the center of a ring $A$.

1. A $u$-demiring is a subsemiring of $A$ that contains $u$ and does not contain $-u$.

2. A $u$-hemiring is a maximal $u$-demiring.

3. We say that a $u$-demiring $D$ is archimedean if $u+n a \in D$ for every $n \in N$ implies $a \in D$.

4. A $u$-demiring $D$ is conic if $|D|=0$, where $|D|=D \cap(-D)$.

5. A $u$-demiring $D$ is $u$-cancellable if $u a \in D$ implies $a \in D$.

6. Let $D$ be a $u$-demiring. We say that $u$ is a $D$-unit if for each $a \in A$ there exists an $n \in N$ such that $n u-a \in D$.

7. If $A, A^{\prime}$ are partially ordered rings with positive cones $D, D^{\prime}$ respectively and if $\theta: A \rightarrow A^{\prime}$ is an order preserving monomorphism, we say that

$$
\theta:(A, D) \rightarrow\left(A^{\prime}, D^{\prime}\right)
$$

is a monomorphism.

Proposition 1. Let $A$ be a ring containing an archimedean u-cancellable $u$ demiring of $A$ such that $u$ is a $D$-unit. Then there exists a compact Hausdorff space $X$ and a monomorphism $(A /|D|, D /|D|) \rightarrow(C(X), P)$, where $P$ is the usual pointwise positive cone on $C(X)$.

Proof. Note that $A=D-D$ since $u \in D$ and $u$ is a $D$-unit; thus, $|D|$ is an ideal in $A$. Since all properties of $D$ as a $u$-demiring of $A$ carry over to $D /|D|$ as a $(u+|D|)$-demiring of $A /|D|$, we may assume $D$ is conic.

If $A$ has identity 1 and $u=1$, this is precisely the content of [1, Theorem 1]. Thus, we assume $A$ has no identity. In the standard way, embed $A$ in a ring $A^{\prime}$ with identity. That is, set $A^{\prime}=\{(n, a): n$ is an integer, $a \in A\}$ with coordinatewise addition and with $(n, a)(m, b)=(n m, n b+m a+a b)$, and define $\theta: A \rightarrow A^{\prime}$ via $a \mapsto(0, a)$. Now set $D^{\prime}=\{(n, a): n \geqq 0$ and $n u+u a \in D\}$. A straightforward verification shows that $D^{\prime}$ is a conic archimedean 1-demiring of $A^{\prime}$ such that $1=(1,0) \in D^{\prime}$ and 1 is a $D^{\prime}$-unit. Hence, the result follows from [1, Theorem 1].

By considering $A=\left\{n x+m x^{2}: n\right.$ and $m$ are integers $\}, D=\left\{n x+m x^{2}: n, m \geqq 0\right\}$, and $u=x+x^{2}$, where $A$ has the usual polynomial addition and multiplication 
except that $x^{3}=0$, we see that the $u$-cancellability hypothesis of Proposition 1 is independent of the other hypotheses. Moreover, it is clear that $D$ must be $u$ cancellable if Proposition 1 is to hold.

Proposition 2. Let $H$ be a u-hemiring of a ring $A$ such that $u$ is an $H$-unit. Then there exists a unique monomorphism $\theta:(A /|H|, H /|H|) \rightarrow\left(R, R^{+}\right)$. If $A /|H|$ is a zero ring, i.e. $(A /|H|)^{2}=0$, then $\theta$ is, of course, only an additive monomorphism.

Proof. One shows that $|H|$ is an ideal in $A$ and that the properties of $H$ in $A$ carry over to $H /|H|$ in $A /|H|$, so we assume $H$ is conic.

If $A^{2}=0$, it is immediate that $H$ is a $u$-hemigroup as defined in [5], whence the result follows from [5, Lemma 5]. Thus, we assume $A^{2} \neq 0$ so that there exist $a, b \in A$ with $a b \neq 0$. Since $u$ is an $H$-unit and $u \in H$, there exists $n \in N$ such that $n u \pm a$, $n u \pm b \in H$. Suppose $u^{2}=0$. Then $\pm u a=u(n u \pm a) \in H$ and $\pm u b \in H$, whence $u a=u b \in|H|=0$. But then $\pm a b=(n u \pm a)(n u+b) \in H$, which gives the contradiction $a b=0$. Hence, $u^{2} \neq 0$. Now suppose $-u^{m} \in H$ for some $m \in N$. Assume $m$ is minimal, so that $-u^{m-1} \notin H$. Then $-u$ belongs to the subsemiring generated by $H$ and $-u^{m-1}$. Since $u,-u^{m} \in H$, it follows that $-u=h_{0}+h_{1}\left(-u^{m-1}\right)+k\left(-u^{m-1}\right)$ for some $h_{0}, h_{1} \in H$ and $k \in N \cup\{0\}$. But multiplication by $u$ then gives the contradiction $-u^{2} \in H$. Thus, $-u^{m} \notin H$ for every $m \in N$. One can then show that $S=\left\{a \in A: u^{n} a \in H\right.$ for some $\left.n \in N\right\}$ is a $u$-demiring of $A$ containing $H$. Hence, $S=H$, proving that $H$ is $u$-cancellable.

Since $H$ is conic and $u$-cancellable, $u^{m} \neq 0$ for every $m \in N$, so that we may consider the ring of quotients $A^{\prime}=\left\{a / u^{n}: a \in A, n \in N\right\}$ with the usual addition and multiplication and with identity $1=u / u$. One checks that $H^{\prime}=\left\{a / u^{n}: a \in H\right\}$ is a conic 1-demiring of $A^{\prime}$ containing 1 and that $a \mapsto u a / u$ is a monomorphism $(A, H) \rightarrow\left(A^{\prime}, H^{\prime}\right)$. Let $x=a / u^{n} \in A^{\prime} \backslash H^{\prime}$. Then $a \notin H$, so that $-u$ belongs to the subsemiring of $A$ generated by $H$ and $a$. Thus, $-u$ is a sum of terms of the form $h_{1}^{n}{ }_{1} a^{m_{1}} h_{2}^{n_{2}} \cdots a^{m_{r}} h_{r^{n}}^{n_{r}}$, so that $-1=-u / u$ is a sum of terms of the form

$$
\frac{u}{u^{2}} \frac{u^{n_{1} m_{1}+1} h_{1}^{n_{1}}}{u} x^{m_{1}} \frac{u^{n_{2} m_{2}+1} h_{2}^{n_{2}}}{u} \cdots x^{m_{r}} \frac{u^{n_{r} m_{r}+1} h_{r}^{n_{r}}}{u}
$$

whence -1 belongs to the subsemiring of $A^{\prime}$ generated by $H^{\prime}$ and $x$. Hence, $H^{\prime}$ is a 1-hemiring of $A^{\prime}$. Now $-u^{2} \notin H$ implies that $-u$ belongs to the subsemiring generated by $H$ and $-u^{2}$. It follows that $u h-u \in H$ where $h=\sum_{i=1}^{r} h_{i} u^{2 i-1}$ $+\sum_{i=1}^{r} n_{i} u^{2 i-1}$ for some $h_{1}, \ldots, h_{r} \in H$ and $n_{1}, \ldots, n_{r}, r \in N \cup\{0\}$. Choose $s \in N$ such that $s u-h \in H$. Then $s u^{2}-u=u(s u-h)+u h-u \in H$, and, by induction, $s^{t} u^{t+1}-u \in H$ for all integers $t \geqq 1$. For each $a / u^{t} \in A^{\prime}$ there exists, by hypothesis, $q \in N$ such that $q u-a u \in H$, whence $q s^{t} 1-a / u^{t}=\left(q\left(s^{t} u^{t+1}-u\right)+q u-a u\right) / u^{t+1} \in H^{\prime}$. Therefore, 1 is an $H^{\prime}$-unit in $A^{\prime}$. The existence of the desired monomorphism thus follows from [3, Proposition 7].

Uniqueness follows from the next proposition.

Proposition 3. If $K$ and $M$ are subrings of $R$ and $\theta: K \rightarrow M$ is an order isomorphism of $K$ onto $M$, then $K=M$ and $\theta$ is the identity map on $K$. 
Proof. First assume $K$ has a least positive element $k$. Then $m=\theta(k)$ is the least positive element of $M$ and a standard argument shows that $K=\{n k: n$ is an integer $\}$ and $M=\{n m: n$ is an integer $\}$. Since $k^{2} \in K, k$ must be an integer. It follows that $k=m$, so that $K=M$ and $\theta$ is the identity.

Now assume that $K$ and hence $M$ have arbitrarily small positive elements, so they are dense in $R$. Suppose there exists $t<1$ in $K$ such that $t \neq \theta(t)$. If $1 \leqq \theta(t)$, then $\theta(t) \leqq \theta\left(t^{2}\right)$, a contradiction. Hence, $t$ and $\theta(t)$ are between 0 and 1 .

Suppose $0<\theta(t)<t<1$. By the density of $K$, there exists $s>1$ in $K$ with $s \theta(t)<1$ $<s t$. Since $\theta(s) \leqq s$ would imply $\theta(s t)=\theta(s) \theta(t) \leqq s \theta(t)<1$, which gives the contradiction $\theta(s t)^{2}<\theta(s t)$, we must have $1<s<\theta(s)$.

We now prove that if $a, b, c, d \in R$ are such that $0<a<b$ and $1<c<d$, then there exist $n, m \in N$ with $c^{n}<m a<m b<d^{n}$. To see this, simply choose $n \in N$ so that $n \log c \geqq \log a$ and $n(\log d-\log c)>1+\log b-\log a$, and let $m \in N$ be least such that $n \log c<\log m+\log a$. Since $\log (j+1)-\log j<1$ for every $j \in N$, we have $\log m+\log a-n \log c<1$. It then follows that $n \log c<\log m+\log a<\log m$ $+\log b<n \log d$. This yields $c^{n}<m a<m b<d^{n}$.

Thus, taking $s=c, \theta(s)=d, \theta(t)=a$, and $t=b$, we obtain $s^{n}<\theta(m t)<m t<\theta\left(s^{n}\right)$ for some $n, m \in N$. Since this clearly leads to a contradiction, we may assume $0<t<\theta(t)<1$, whence $0<\theta^{-1}(t)<t<1$. But $M$ is also dense and $\theta^{-1}$ is an order isomorphism, so that we can apply the above argument to $M$ and $\theta^{-1}$ and again obtain a contradiction. Therefore, $\theta(t)=t$.

It follows that $\theta$ fixes a subring of $K$ that is dense in $R$, so a standard argument shows that $\theta$ fixes $K$.

Remark. The existence proof can also be established by proving that $H$ is archimedean so that Proposition 1 yields a compact space $X$ and a monomorphism $\Psi:(A, H) \rightarrow(C(X), P)$, and then using the maximality of $H$ to show that $\Psi$ maps into the constant functions on $X^{*}=\{x \in X: \Psi(a)(x) \neq 0$ for some $a \in A\}$.

\section{Embedding in a division ring.}

Definitions. 1. We say that a $u$-demiring $D$ is left central if for each $d \in D$ and $a \in A$ there exists $d^{\prime} \in D$ such that $a d=d^{\prime} a$.

2. A subset of a ring is right (resp. left) cancellable if whenever $a b \in S$ and $b$ (resp. $a$ ) is a nonzero element of $S, a$ (resp. $b$ ) belongs to $S$.

3. For $D$ a $u$-demiring, $u$ is a weak $D$-unit if for each $a \in D$ there exists $n \in N$ with $n u-a \in D$.

Note that if $H$ is a left central $u$-hemiring of a ring and $x \notin H$, then $-u=\sum_{i=0}^{m} h_{i} x^{i}$ $+\sum_{i=1}^{m} n_{i} x$ for some $h_{0}, \ldots, h_{m} \in H$ and $n_{1}, \ldots, n_{m}, m \in N \cup\{0\}$.

LeMma 1. Let $H$ be a left central u-hemiring of a ring $A$ such that $u \in H$ and $-u^{2} \notin H$, and let $M=H \backslash|H|$. Then the following statements hold:

(a) $M$ is a multiplicative subset of $A$;

(b) $M$ is left and right cancellable;

(c) if $H$ is conic, then $A$ has no proper right or left zero divisors. 
Proof. First note that since $-u^{2} \notin H$, one can show that $H$ is $u$-cancellable as in the proof of Proposition 2.

(a) Suppose $x, y \in M$ with $x y \in|H|$. Then $-x \notin H$, so that $-u=\sum_{i=0}^{m} h_{i}(-x)^{i}$ $+\sum_{i=1}^{m} n_{i}(-x)^{i}$ for some $h_{0}, \ldots, h_{m} \in H$ and $n_{1}, \ldots, n_{m}, m \in N \cup\{0\}$. Thus,

$$
-u y=h_{0} y-\left(\sum_{i=1}^{m} h_{i}(-x)^{i-1}\right)(x y)-n_{1} x y-\left(\sum_{i=2}^{m} n_{i}(-x)^{i-1}\right)(x y) \in H
$$

since $|H|$ is clearly an ideal in $H-H$. Hence, $-y \in H$, giving the contradiction $y \in|H|$. Thus, $x y \in M$.

(b) Suppose $a b \in M, b \in M$, but $a \notin M$. Since $a \in|H|$ would imply the contradiction $a b \in|H|$, we have $a \notin H$. Then $-u=\sum_{i=0}^{m} h_{i} a^{i}+\sum_{i=1}^{m} n_{i} a^{i}$ for some $h_{0}, \ldots, h_{m} \in H$ and $n_{1}, \ldots, n_{m}, m \in N \cup\{0\}$. Now by the left central hypothesis and induction, one shows that for $1 \leqq i \leqq m, a^{i} b^{m}=g_{i} b^{m-1}$ for some $g_{i} \in H$. Thus, $-u b^{m}$ is seen to belong to $H$, whence $-b^{m} \in H$. Hence, $b^{m} \in|H|$, which contradicts part (a). Therefore, $a \in M$, proving that $M$ is right cancellable. The proof of left cancellability is similar.

(c) Assume $H$ is conic, $a b=0$, and $b \neq 0$. If $b \in H$, then $a \in H$ since otherwise we would get the contradiction $b^{m} \in|H|=0$ as in part (b). Likewise, since $(-a) b=0$ we also have $-a \in H$, whence $a \in|H|=0$.

If $b \notin H$, then $-u=\sum_{j=0}^{n} h_{j} b^{j}+\sum_{j=1}^{n} m_{j} b^{j}$ for some $h_{0}, \ldots, h_{n} \in H$ and $m_{1}, \ldots, m_{n}, n \in N \cup\{0\}$. Thus, $-a u=a h_{0}$, so $a\left(h_{0}+u\right)=0$. Since $-u \notin H, h_{0}+u$ is a nonzero element of $H$. Hence, $a=0$ as above.

Similarly, if $b a=0$ and $b \neq 0$, then $a=0$.

A slight generalization of a well-known result of $\mathrm{O}$. Ore [7, Theorem 1] is now given.

LEMMA 2. Let $A$ be a ring with a nonempty right cancellable multiplicative subset $M$ consisting of elements that are neither left nor right zero-divisors in A. Assume that for every $a \in A, b \in M$ there are $a_{1} \in A, b_{1} \in M$ such that $b_{1} a=a_{1} b$. Then there exist a ring $A^{\prime}$ with identity and a monomorphism $\theta: A \rightarrow A^{\prime}$ such that for each $b \in M, \theta(b)$ has a two-sided inverse in $A^{\prime}$. Moreover, each element of $A^{\prime}$ is of the form $\theta(b)^{-1} \theta(a)$.

Proof. Note that if $b, d \in M$ and we choose $d_{1} \in A, b_{1} \in M$ such that $d_{1} b=b_{1} d$, then the right cancellability of $M$ implies that $d_{1} \in M$. Also note that if $n a=m b$ and $n_{1} a=m_{1} b$ for some $b, n_{1} \in M$ and $n, a, m, m_{1} \in A$, then by choosing $r \in M$ and $s \in A$ such that $r n=s n_{1}$, we obtain $r m b=r n a=s n_{1} a=s m_{1} b$, whence $r m=s m_{1}$.

Ore's proof now goes through if one notes at each step that the appropriate elements belong to $M$ : Let $S=A \times M$ and define $(a, b) \equiv(c, d)$ if $d_{1} a=b_{1} c$, where $d_{1}, b_{1} \in M$ are such that $d_{1} b=b_{1} d$. Show that $\equiv$ is a well-defined equivalence relation on $S$. Denote the class containing $(a, b)$ by $a / b$ and let $A^{\prime}=\{a / b:(a, b) \in S\}$; define $a / b+c / d=\left(d_{1} a+b_{1} c\right) / b_{1} d$ where $b_{1}, d_{1} \in M$ are such that $d_{1} b=b_{1} d$, and define $(a / b)(c / d)=a_{1} c / g b$ where $g \in M$ and $a_{1} \in A$ are such that $g a=a_{1} d$. Show that 
$A^{\prime}$ is a ring with identity $1=b / b$ for any $b \in M, a \mapsto b a / b$ is a monomorphism $\theta: A \rightarrow A^{\prime}, b / b^{2}$ is a two-sided inverse of $\theta(b)$ for any $b \in M$, and $a / b=\theta(b)^{-1} \theta(a)$ for any $a / b \in A^{\prime}$.

THEOREM 1. Let $H$ be a conic left central u-hemiring of a ring $A$ such that $u \in H$, $u$ is a weak $H$-unit, and $H^{2} \neq 0$. Then there exist a conic 1-hemiring $H^{\prime}$ in a division ring $A^{\prime}$, a monomorphism $(A, H) \rightarrow\left(A^{\prime}, H^{\prime}\right)$, and a monomorphism $\left(H^{\prime}-H^{\prime}, H^{\prime}\right)$ $\rightarrow R$, where $F=H^{\prime}-H^{\prime}$ is a subfield of $A^{\prime}$ over which $A^{\prime}$ is algebraic. Moreover, if $A$ is commutative, so is $A^{\prime}$.

Proof. From the fact that $u$ is a weak $H$-unit, one shows $u$ is an $H$-unit in the ring $H-H$. Since $H^{2} \neq 0$, Proposition 2 yields a monomorphism $(H-H, H) \rightarrow R$. In particular, $u^{2} \neq 0$, every nonzero element of $H$ is a weak $H$-unit, and $H$ is commutative.

By Lemma $1, M=H \backslash\{0\}$ is a right cancellable multiplicative set consisting of regular elements of $A$. Let $a \in A$ and $b \in M$. Then $a b=h a$ for some $h \in M$. Therefore Lemma 2 says that $A^{\prime}=\{a / b: a \in A, b \in M\}$ is a ring and $a \mapsto b a / b$ is a monomorphism $\theta: A \rightarrow A^{\prime}$ such that $\theta(b)$ is invertible in $A^{\prime}$ for each $b \in M$. Since $H$ is left central, equality, addition, and multiplication now have the following simpler forms:

$$
\begin{aligned}
a / b & =c / d \quad \text { if } d_{1} a=b c, \text { where } d_{1} \in M \text { is such that } d_{1} b=b d ; \\
a / b+c / d & =\left(d a+b_{1} c\right) / b_{1} d, \text { where } d b=b_{1} d, b_{1} \in M ; \\
(a / b) \cdot(c / d) & =a c / g b, \quad \text { where } g a=a d, g \in M .
\end{aligned}
$$

Let $H^{\prime}=\left\{a / b \in A^{\prime}: a / b=c / d\right.$ for some $c / d \in A^{\prime}$ with $\left.c \in H\right\}$. Then the left cancellability of $M$ implies $H^{\prime}=\left\{a / b \in A^{\prime}: a \in H\right\}$. From this, one easily checks that $H^{\prime}$ is a conic 1-demiring of $A^{\prime}$ such that $1 \in H^{\prime}$, every nonzero element of $H^{\prime}$ has an inverse in $H^{\prime}$, and $\theta:(A, H) \rightarrow\left(A^{\prime}, H^{\prime}\right)$ is a monomorphism.

Now assume $x / y \in A^{\prime} \backslash H^{\prime}$. Then $x \notin H$, so that $-u=\sum_{i=0}^{m} h_{i} x^{i}+\sum_{i=1}^{m} n_{i} x^{i}$ for some $h_{i}$ 's $\in H$ and $n_{i}$ 's $\in N \cup\{0\}$. By definition of the operations in $A^{\prime}$, it follows that

$$
\sum_{i=1}^{m} \frac{u h_{i}}{u} \frac{f_{i}}{u}\left(\frac{x}{y}\right)^{i}+\sum_{i=1}^{m} \frac{n_{i} u}{u} \frac{f_{i}}{u}\left(\frac{x}{y}\right)^{i}=\frac{-u}{u}=-1
$$

where $\left(u h_{i} / u\right)\left(f_{i} / u\right)$ and $\left(n_{i} u / u\right)\left(f_{i} / u\right)$ belong to $H^{\prime}$ for every $i$. Hence, we see that $H^{\prime}$ is a 1 -hemiring of $A^{\prime}$.

Let $a / b \in A^{\prime}$. As noted earlier, each element of $H$ is a weak $H$-unit, so there exists $n \in N$ such that $n b-a \in H$, whence $n 1-a / b=u(n b-a) / u b \in H^{\prime}$. Therefore, 1 is a weak $H^{\prime}$-unit in $A^{\prime}$. From Proposition 2, it then follows that there exists a monomorphism $\left(H^{\prime}-H^{\prime}, H^{\prime}\right) \rightarrow R$. Thus, $F=H^{\prime}-H^{\prime}$ is a subfield of $A^{\prime}$.

If $x / y \in A^{\prime}$ and $a / b \in H^{\prime}$, so that $a \in H$, then there exists $a_{1} \in H$ such that $x a=a_{1} x$. Now $(x / y)(a / b)=x a / g y$, where $g \in M$ and $g x=x b$. Thus, the commutativity of $H$ yields

$$
\frac{x}{y} \frac{a}{b}=\frac{x a}{g y}=\frac{a_{1} x}{y g}=\frac{a_{1}}{g} \frac{x}{y}
$$


Since $a_{1} / g \in H^{\prime}, H^{\prime}$ is therefore left central in $A^{\prime}$.

It follows that each $x \in A^{\prime} \backslash H^{\prime}$ satisfies $-1=\sum_{i=0}^{n} h_{i} x^{i}$ for some $h_{i}$ 's $\in H^{\prime}$, and thus has $-\left(1+h_{0}\right)^{-1} \sum_{i=0}^{n} h_{i} x^{i}$ as left inverse. Multiplying on the left by $x$ yields $-x=\sum_{i=0}^{n} h_{i}^{\prime} x^{i+1}$ for some $h_{0}^{\prime}, \ldots, h_{n}^{\prime} \in H$, so $\left(1+\sum_{i=0}^{n} h_{i}^{\prime} x^{i}\right) x=0$, whence $-1-h_{0}^{\prime}$ $=\sum_{i=1}^{n} h_{i}^{\prime} x^{i}$ by Lemma $1(\mathrm{c})$. Hence, $x$ has $\left(\sum_{i=1}^{n} h_{i} x^{i}\right)\left(-1-h_{0}^{\prime}\right)^{-1}$ as right inverse. It follows that $A^{\prime}$ is a division ring each element of which satisfies a polynomial over the field $F$. Since $H^{\prime}$ is left central, $a F a^{-1} \subseteq F$ for every nonzero $a \in A$. Thus, the Cartan-Brauer-Hua Theorem [4, p. 186] implies that either $A=F$ or $F$ is contained in the center of $A$. The result follows in either case.

That $A^{\prime}$ is commutative if $A$ is commutative follows from the definition of multiplication in $A^{\prime}$.

Henceforth we write $C$ for the field of complex numbers and $Q$ for the division ring of real quaternions. As corollaries we have the following two theorems.

THEOREM 2. Let $A$ be a commutative ring containing a conic u-hemiring $H$ such that $u$ is a weak $H$-unit, $u \in H$, and $H^{2} \neq 0$. Then there exists a monomorphism $\theta:(A, H) \rightarrow\left(C, R^{+}\right)$which is unique on $H-H$. Moreover, $\theta$ maps into $R$ iff $u$ is an H-unit.

Proof. By Theorem 1, there exist a field $A^{\prime}$, a monomorphism

$$
\theta_{1}:(A, H) \rightarrow\left(A^{\prime}, H^{\prime}\right)
$$

and a monomorphism

$$
\theta_{2}:\left(H^{\prime}-H^{\prime}, H^{\prime}\right) \rightarrow\left(R, R^{+}\right),
$$

where $H^{\prime}-H^{\prime}$ is a subfield of $A^{\prime}$ and $A^{\prime}$ is algebraic over $H^{\prime}-H^{\prime}$. Since $C$ is algebraically closed, $\theta_{2}$ extends to a monomorphism $\theta_{3}:\left(A^{\prime}, H^{\prime}\right) \rightarrow\left(C, R^{+}\right)$. Hence, $\theta=\theta_{3} \theta_{1}$ is a monomorphism $(A, H) \rightarrow\left(C, R^{+}\right)$.

By Proposition 3, $\theta$ is unique on $H-H$.

If $u$ is an $H$-unit, $H-H=A$ so $\theta: A \rightarrow R$. The converse is also clear.

THEOREM 3. The semiring $R^{+}$is a left central 1-hemiring of a ring $A$ iff $A$ is isomorphic to one of $R, C$, or $Q$.

Proof. Necessity follows immediately from Theorem 1 and Frobenius' Theorem on real division algebras. Sufficiency is contained in the following example.

EXAMPLE. We generalize an example stated without proof in [2, Example 3]. Let $H$ be a subsemiring of $R^{+}$. Let $A$ be any subring of $Q$ whose elements all have the form $a+b i+c j+d k$ with $a, b, c, d \in H-H$. We show that $H$ is a 1-hemiring of $A$. Note that $H$ is also conic and left central and that 1 is a weak $H$-unit.

Let $x=a+b i+c j+d k \in A \backslash H$ and let $H^{\prime}$ be the subsemiring of $A$ generated by $H$ and $x$. It suffices to show $-1 \in H^{\prime}$.

Case I. $a=0$. Then $x^{2} \in-H$, so there exists $n \in N$ such that $n x^{2} \leqq-1$. Hence, $-1=\left(-n x^{2}-1\right)+n x^{2} \in H^{\prime}$. 
Case II. $a<0$. If $x \neq a$, an application of Case I to $x+(-a)$ again yields $-1 \in H^{\prime}$. If $x=a$, choose $m$ such that $m x \leqq-1$. Then $-1=(-m x-1)+m x \in H^{\prime}$.

Case III. $a>0$. Then one of the remaining coefficients, say $b$, must be nonzero. The proof is similar if either $c$ or $d$ is nonzero. Let $y=a+b i$, and set $x_{0}=x-y+c j$ $+d k$. Then $x_{0}^{2}=y^{2}-c^{2}-d^{2}+t_{1}$, where $t_{1}=c_{1} j+d_{1} k$ for some $c_{1}, d_{1} \in H-H$. Thus, $x_{1}=y^{2}+t_{1}=x^{2}+c^{2}+d^{2} \in H^{\prime}$. Repeating this, we see that $x_{2}=x_{1}^{2}+c_{1}^{2}+d_{1}^{2}$ $=y^{4}+t_{2}$, where $t_{2}$ has the form $c_{2} j+d_{2} k$ for some $c_{2}, d_{2} \in H-H$, whence $x_{2} \in H^{\prime}$. Continuing inductively, we obtain a sequence $\left\{x_{n}\right\}$, where $x_{n}=y^{2^{n}}+t_{n} \in H^{\prime}$ and $t_{n}=c_{n} j+d_{n} k$ for some $c_{n}, d_{n} \in H-H$. There exists $n \in N$ such that $y^{2^{n}}$ has negative real part, so $x_{n}$ has this same negative real part. Hence, Case II shows that -1 belongs to the subsemiring generated by $x_{n}$ and $H$, and therefore $-1 \in H^{\prime}$.

\section{REFERENCES}

1. D. W. Dubois, A note on David Harrison's theory of preprimes, Pacific J. Math. 21 (1967), 15-19. MR 35 \#103.

2. - Second note on David Harrison's theory of preprimes, Pacific J. Math. 24 (1967), 57-68. MR 36 \#5049.

3. D. K. Harrison, Finite and infinite primes for rings and fields, Mem. Amer. Math. Soc. No. 68 (1966). MR 34 \#7550.

4. N. Jacobson, Structure of rings, rev. ed., Amer. Math. Soc. Colloq. Publ., vol. 37, Amer. Math. Soc., Providence, R. I., 1968.

5. C. W. Kohls, Representation of abelian groups and rings by families of real-valued functions, Proc. Amer. Math. Soc. 25 (1970), 86-92.

6. J. L. Krivine, Anneaux preordonnés, J. Analyse Math. 12 (1964), 307-326. MR 31 \#213.

7. O. Ore, Linear equations in noncommutative fields, Ann. of Math. 32 (1931), 463-477.

8. R. S. Palais, The classification of real division algebras, Amer. Math. Monthly 75 (1968), 366-368. MR 37 \#4119.

SYRACUSE UNIVERSITY,

Syracuse, New York 13210 